\title{
Weight change and risk of cardiovascular disease among adults with type 2 diabetes: more than 14 years of follow-up in the Tehran Lipid and Glucose Study
}

Seyyed Saeed Moazzeni ${ }^{1}$, Reyhane Hizomi Arani ${ }^{1}$, Niloofar Deravi ${ }^{1,2}$, Mitra Hasheminia ${ }^{1}$, Davood Khalili ${ }^{1,3}$, Fereidoun Azizi $^{4}$ and Farzad Hadaegh ${ }^{1 *}$ (D)

\begin{abstract}
Background: To examine the impact of weight change on incident cardiovascular disease and coronary heart disease (CVD/CHD) among an Iranian population with type 2 diabetes mellitus (T2DM).

Methods: The study population included 763 participants with T2DM aged $\geq 30$ years without a history of CVD and cancer at baseline. Two weight measurements done at baseline and about 3 years later. Based on their weight change, they categorized into: $>5 \%$ loss, 3-5\% loss, stable $( \pm<3 \%), 3-5 \%$ gain, $>5 \%$ gain. Participants were then followed for incident CVD/CHD annually up to 20 March 2018. Multivariable Cox proportional hazard models, adjusted for age, sex, body mass index, educational level, current smoking, glucose-lowering drug use, family history of CVD, hypertension, hypercholesterolemia, chronic kidney disease, and fasting plasma glucose (FPG) were applied to estimate the hazard ratios (HRs) and 95\% confidence intervals (Cls) of weight change categories for incident CVD/CHD, considering stable weight as reference.
\end{abstract}

Results: After the weight change measurement, during a median follow-up of 14.4 years, 258 CVD and 214 CHD occurred. Over $5 \%$ weight gain was associated with reduced risks of CVD and CHD development by the HRs of 0.70 [95\% Cl 0.48-1.01; P-value: 0.058] and 0.61 [0.40-0.93], respectively, in multivariable analysis. After further adjustment for FPG change, the HRs of weight gain > 5\% were attenuated to 0.75 [0.51-1.10; P-value: 0.138] and 0.66 [043-1.01; P-value: 0.053] for incident CVD and CHD, respectively. The effect of weight loss $>5 \%$ was in opposite direction among those older versus younger than 60 years; with suggestive increased risk (not statistically significant) of incident CHD/ CVD for the older group. Moreover, weight gain $>5 \%$ significantly reduced the risk of CHD only among those older than 60 years (P-value for interaction $<0.2$ ). Furthermore, weight gain $>5 \%$ had an association with lower risk of CVD and CHD among sulfonylurea users (0.56 [0.32-0.98] for CVD and 0.54 [0.29-0.99] for CHD).

Conclusions: Our results with a long-term follow-up showed that weight gain > 5\% was associated with better CVD/ CHD outcomes among Iranian participants with T2DM, especially older ones. Moreover, we did not find an unfavorable impact on incident CVD/CHD for sulfonylurea-induced weight gain.

\footnotetext{
*Correspondence: fzhadaegh@endocrine.ac.ir

1 Prevention of Metabolic Disorders Research Center, Research Institute for Endocrine Sciences, Shahid Beheshti University of Medical Sciences, No. 24, Parvaneh Street, Velenjak, P.O. Box: 19395-4763, Tehran, Iran

Full list of author information is available at the end of the article
}

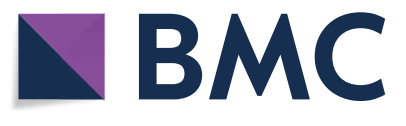

(c) The Author(s) 2021. This article is licensed under a Creative Commons Attribution 4.0 International License, which permits use, sharing, adaptation, distribution and reproduction in any medium or format, as long as you give appropriate credit to the original author(s) and the source, provide a link to the Creative Commons licence, and indicate if changes were made. The images or other third party material in this article are included in the article's Creative Commons licence, unless indicated otherwise in a credit line to the material. If material is not included in the article's Creative Commons licence and your intended use is not permitted by statutory regulation or exceeds the permitted use, you will need to obtain permission directly from the copyright holder. To view a copy of this licence, visit http://creativeco mmons.org/licenses/by/4.0/. The Creative Commons Public Domain Dedication waiver (http://creativecommons.org/publicdomain/ zero/1.0/) applies to the data made available in this article, unless otherwise stated in a credit line to the data. 
Keywords: Diabetes mellitus, type 2, Cardiovascular diseases, Coronary disease, Body weight changes, Sulfonylurea compounds

\section{Background}

The Middle East and North Africa (MENA) region had the second-highest prevalence of type 2 diabetes mellitus (T2DM) in 2017 globally (i.e., 10.8\%), with an increasing pattern during the past three decades [1]. In 2011, nearly 4.5 million adults were living with diabetes in Iran, and it is estimated that 9.2 million Iranian adults will be affected by diabetes by the year 2030 [2]. This unceasing significant growth reveals a high burden of T2DM in Iran, particularly when considering its complications [2-4]. T2DM is the leading cause of cardiovascular disease (CVD) and mortality events among Iranian populations. Previously, we found that the population attributable fraction of T2DM for incident CVD events was $14 \%$ among Tehranian adults [5].

To improve hyperglycemia and cardiometabolic risk factors, intentional weight loss was recommended to overweight and obese patients with T2DM; however, the effect of weight loss on incident CVD events is less clear [6-8]. Moreover, unintentional weight loss is a marker of major health problems and sarcopenia; it can also be associated with increased morbidity and mortality, especially among older individuals [9]. Appropriate interventions to maintain a stable weight were also recommended to positively influence health outcomes, especially CVD outcomes, among patients with T2DM $[10,11]$. Glucose-lowering drugs (GLDs) also can have effects on weight by inducing weight gain or weight loss among patients with T2DM [12].

There are few investigations about the impact of weight change on the risk of CVD events among populations with T2DM, with heterogenic designs and findings. A meta-analysis in 2018 reported that weight loss, but not weight gain, increased CVD mortality among individuals with T2DM; however, there was significant heterogeneity between the included studies $\left(\mathrm{I}^{2}=98 \%\right)$ [13]. Results of the recent studies on this issue also remained inconsistent. Data from the ORIGIN trial and the Anglo-Danish-Dutch Study of Intensive Treatment in People with Screen-Detected Diabetes in Primary Care (ADDITION)-Cambridge trial showed significant protection for weight loss among participants with T2DM [14, 15]; however, the Action in Diabetes and Vascular disease: preterAx and diamicroN-MR Controlled Evaluation (ADVANCE) trial showed a significantly increased risk of CVD among participants with weight loss [16]. Moreover, results from other studies were not statistically significant for the association of weight loss with CVD [17-19]. Similar to weight loss, findings for the effect of weight gain on CVD events were inconclusive among populations with T2DM, in which adverse [16, 20], protective [15], and neutral $[18,19]$ effects have been reported for weight gain all together.

It also should be noted that previous studies on this issue were mainly limited to the US, European, and East Asian populations. Consequently, in the present study, we examined the impact of 3-year weight change on incident CVD/coronary heart disease (CHD) events among an Iranian population with T2DM during more than a decade of follow-up. Data from the oldest cohort of the MENA region, the Tehran Lipid and Glucose Study (TLGS), was used in this observational study.

\section{Methods}

\section{Study design and study population}

The TLGS is a prospective cohort study conducted on a general population in district 13 of Tehran. The first and second registration phases were on January 31, 1999July 03, 2001 and October 20, 2001-September 22, 2005, respectively, and the cohort examinations were repeated every 3 years. The TLGS had an original aim of assessment the prevalence and incidence of non-communicable diseases and their risk factors. Also, the impact of changes in lifestyle factors was determined in this cohort. The design, measurement methods, and enrollment strategy of the study have been explained in detail by Azizi et al. [21]

From a total of 9558 participants aged $\geq 30$ years, 1525 participants were individuals with T2DM. Firstly, we excluded 249 and 11 individuals with prevalent CVD and cancer at baseline, respectively, leading to 1265 participants. Those with missing data on weight measurement at baseline and first follow-up visit and missing data on relevant covariates were also excluded $(n=459)$. No follow-up information was another reason for exclusion $(n=43)$. Finally, we included 763 eligible subjects with T2DM (635 subjects from phase I and 128 new subjects from phase II) in our analysis.

As shown in Fig. 1, for participants who were enrolled at phase I (baseline measurement), after about 3 years, phase II was set for follow-up measurement of weight. Moreover, for those enrolled at phase II (baseline measurement), follow-up measurement of weight was done at phase III (2005-2008). After these two weight measurements for weight change calculation, participants were 


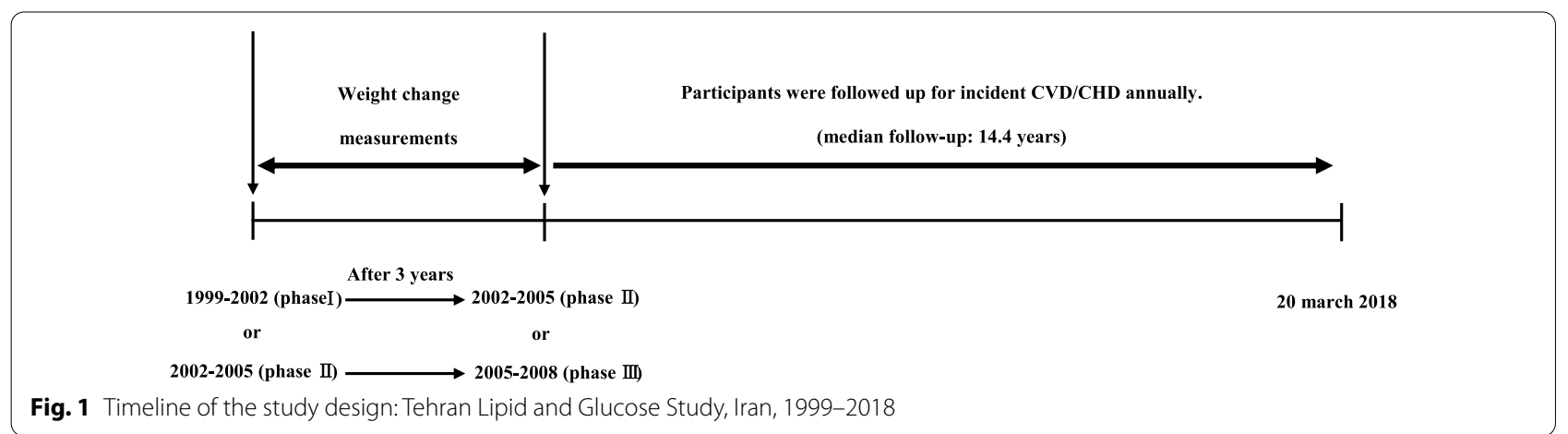

followed-up annually for incident CVD/CHD up to 20 March 2018.

\section{Clinical and laboratory measurements}

Demographic data, medication use, past medical history, family history of premature CVD, smoking habits, and educational level were acquired by validated and interviewer-administered questionnaires at visits. Weight was recorded by a digital scale to the nearest $100 \mathrm{~g}$ while participants had minimal clothes without shoes. Also, we assessed height without shoes in a standing position. Body mass index (BMI) was considered as weight in kilograms divided by height in meters squared. After $15 \mathrm{~min}$ of rest, systolic blood pressure (SBP) and diastolic blood pressure (DBP) were considered the mean of the two physician-measured blood pressure on the right arm using a standard sphygmomanometer. Morning blood samples were collected from all participants after at least $12 \mathrm{~h}$ of fasting. Participants without a history of using GLDs took orally 82.5 g glucose monohydrate solution (equivalent to $75 \mathrm{~g}$ anhydrous glucose), and their blood sample was taken after $2 \mathrm{~h}$ to assess 2 -h post-challenge plasma glucose (2h-PCPG). The measurements of fasting plasma glucose (FPG), 2h-PCPG, total cholesterol, and serum creatinine were performed by standard methods, as previously explained [21].

\section{Definition of terms}

In our study, T2DM was defined as one of these criteria: (a) $\mathrm{FPG} \geq 7 \mathrm{mmol} / \mathrm{L}$; (b) $2 \mathrm{~h}-\mathrm{PCPG} \geq 11.1 \mathrm{mmol} / \mathrm{L}$; (c) taking any GLDs; (d) self-reports of physician-diagnosed diabetes not on GLDs. Moreover, SBP $\geq 140 \mathrm{mmHg}$ or $\mathrm{DBP} \geq 90 \mathrm{mmHg}$ or use of antihypertensive drugs was defined as hypertension. Also, using lipid-lowering drugs or having total cholesterol of $\geq 5.18 \mathrm{mmol} / \mathrm{L}$ was described as hypercholesterolemia. Glomerular filtration rate (GFR) was estimated by the Modification of Diet in Renal Disease (MDRD) study equation [22]. Chronic kidney disease (CKD) was defined as estimated GFR lower than $60 \mathrm{~mL} / \mathrm{min} / 1.73 \mathrm{~m}^{2}$. Educational levels were categorized into three groups: less than 6 years, between 6 and 12 years, and more than 12 years of formal education. We divided our participants based on the smoking status into two groups of current smokers and former/never smokers. We considered the positive family history of premature CVD in participants who have any history of stroke or CHD in female first-degree relatives $<65$ years or male first-degree relatives $<55$ years.

Weight change was calculated as: $\frac{\text { Follow-up measurement-Baseline measurement }}{\text { Baseline measurement }} \times 100$. Then participants were categorized into five groups based on 3 -year weight change percentage: (i) more than $5 \%$ weight loss; (ii) $3 \%$ to $5 \%$ weight loss, (iii) less than $3 \%$ weight change (reference group: stable); (iv) $+3 \%$ to $+5 \%$ weight gain; (v) more than $5 \%$ weight gain, as recommended by Stevens et al. [23].

\section{Outcome assessment}

Details of CVD/CHD data collection have been explained elsewhere [21]. Briefly, a trained nurse interviewed all participants through an annual phone call for any cardiovascular events. Furthermore, any reported event followed by a trained physician through home visits for data collection from medical documents or death certifications (in the cases of mortality). Finally, the outcome committee of the TLGS, consist of needed professionals such as an internist, an endocrinologist, a cardiologist, and an epidemiologist, evaluated the outcome data and adjudicated events. CHD included cases of certain myocardial infarction (MI) [diagnosed by electrocardiogram (ECG) and biomarkers including CK, CK-MB, CK-MBm, troponin (cTn), and myoglobin], probable MI [distinguished by positive ECG findings and cardiac symptoms or positive ECG findings with equivocal biomarkers], unstable angina pectoris [developed new cardiac symptoms or showed changing symptom patterns and positive ECG findings with normal biomarkers] angiographicproven $\mathrm{CHD}$ [defined as $\geq 50 \%$ stenosis in at least one 
major coronary vessel], and cardiac death [any hospital death related to CHD based on the foregoing criteria or sudden cardiac death due to cardiac disease events less than $1 \mathrm{~h}$ after the beginning of symptoms based on verbal autopsy files outside of the hospital]. CVD was considered a composite measure of any CHD, fatal or non-fatal stroke [defined as a rapidly developing new focal or global neurological deficit lasting $\geq 24 \mathrm{~h}$, and death due to cerebrovascular origin.

\section{Statistical analyses}

Comparing baseline characteristics among respondents (study participants) versus non-respondents (including those with missing data of covariates or those without any follow-up data) was performed using Student's $t$-test for continuous variables and chi-square test for categorical variables.

Baseline characteristics across weight change categories are illustrated as mean \pm standard deviation (SD) for continuous variables and number (\%) for categorical variables.

The multivariable Cox proportional regression analysis was applied to evaluate the association of weight change categories with incident $\mathrm{CVD} / \mathrm{CHD}$ by reporting Hazard ratios (HRs) with 95\% confidence intervals (CIs) in two models: Model 1: adjusted for age and sex; Model 2: further adjusted for BMI, educational level, current smoking (at first follow-up), GLDs use (at baseline or first followup), family history of premature CVD, hypertension, hypercholesterolemia, CKD, and FPG.

To address the low power and possibility of bias caused by missing data, as a sensitivity analysis, we examined the impact of weight change on incident CVD/CHD with imputed baseline and first follow-up missing data for covariates using stochastic single imputation with predictive mean matching (PMM) $[24,25]$.

Interactions of weight change categories with age groups ( $\geq 60$ years versus $<60$ years), sex (men versus women), BMI groups ( $\geq 30 \mathrm{~kg} / \mathrm{m}^{2}$ versus $\left.<30 \mathrm{~kg} / \mathrm{m}^{2}\right)$, and GLDs use (yes versus no) were checked by the log-likelihood ratio test in Model 2 and HRs with 95\% CIs calculated for each subgroup.

Time to event was considered as the time of censoring or the outcome (incident CVD/CHD) occurring, whichever came first. We censored subjects in the case of leaving the district, lost to follow-up, or being without any event in the study until 20 March 2018.

Using the Schoenfeld residual test, the proportionality in the Cox model was evaluated; all proportionality assumptions were appropriate. STATA version 14 (StataCorp LP, College Station, Texas) was employed for statistical analyses. A P-value of $<0.05$ and P-value for interaction of $<0.2$ was considered to be statistically significant.

\section{Results}

As presented in Additional file 1: Table S1, there was no significant difference between respondents and nonrespondents except that respondents were about 2 years younger. The study population included 763 individuals (300 men) with T2DM. The mean age was 53.6 (SD: 11.0) years at baseline. Moreover, 352 subjects (46\% of the total population) had been on GLDs at baseline or first followup. As shown in Fig. 2, 304 participants (86.4\% of total GLD users) had used sulfonylureas only or in combination with metformin and/or insulin. The baseline and the first follow-up characteristics of the participants across weight change categories are shown in Table 1 . About $45 \%$ of our participants had a stable weight $(-3 \%$ to $+3 \%$ ) during the first three years of follow-up. Moreover, $16.1 \%$ and $17.3 \%$ of the total participants had more than $5 \%$ weight loss and weight gain, respectively. Weight gain $>5 \%$ was associated with reduced FPG level after 3 years, but having a weight loss $>3 \%$ was accompanied by elevated FPG after 3 years.

During a median follow-up of 14.4 years after the weight change measurement [interquartile range: 12.115.5], 258 CVD (CHD:214) occurred. Multivariable HRs and 95\% CIs of association between weight change categories and incident CVD/CHD events are presented in Tables 2 and 3. Compared to those with stable weight, gaining weight more than $5 \%$ had sex- and age-adjusted HRs of 0.71 [95\% CI 0.49-1.03; P-value: 0.075] for incident CVD and 0.62 [0.41-0.94] for incident CHD. After

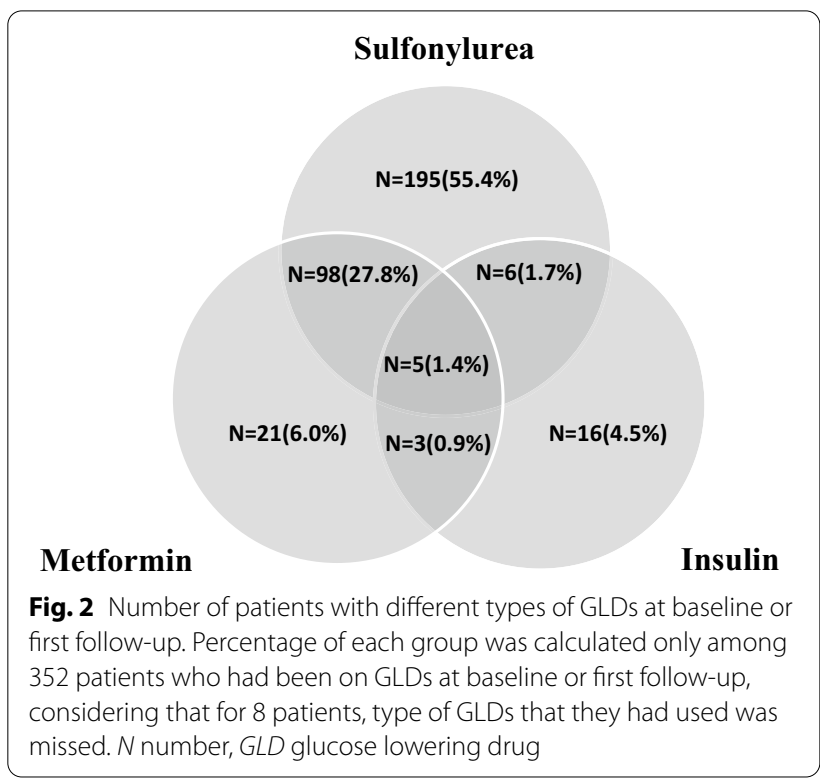




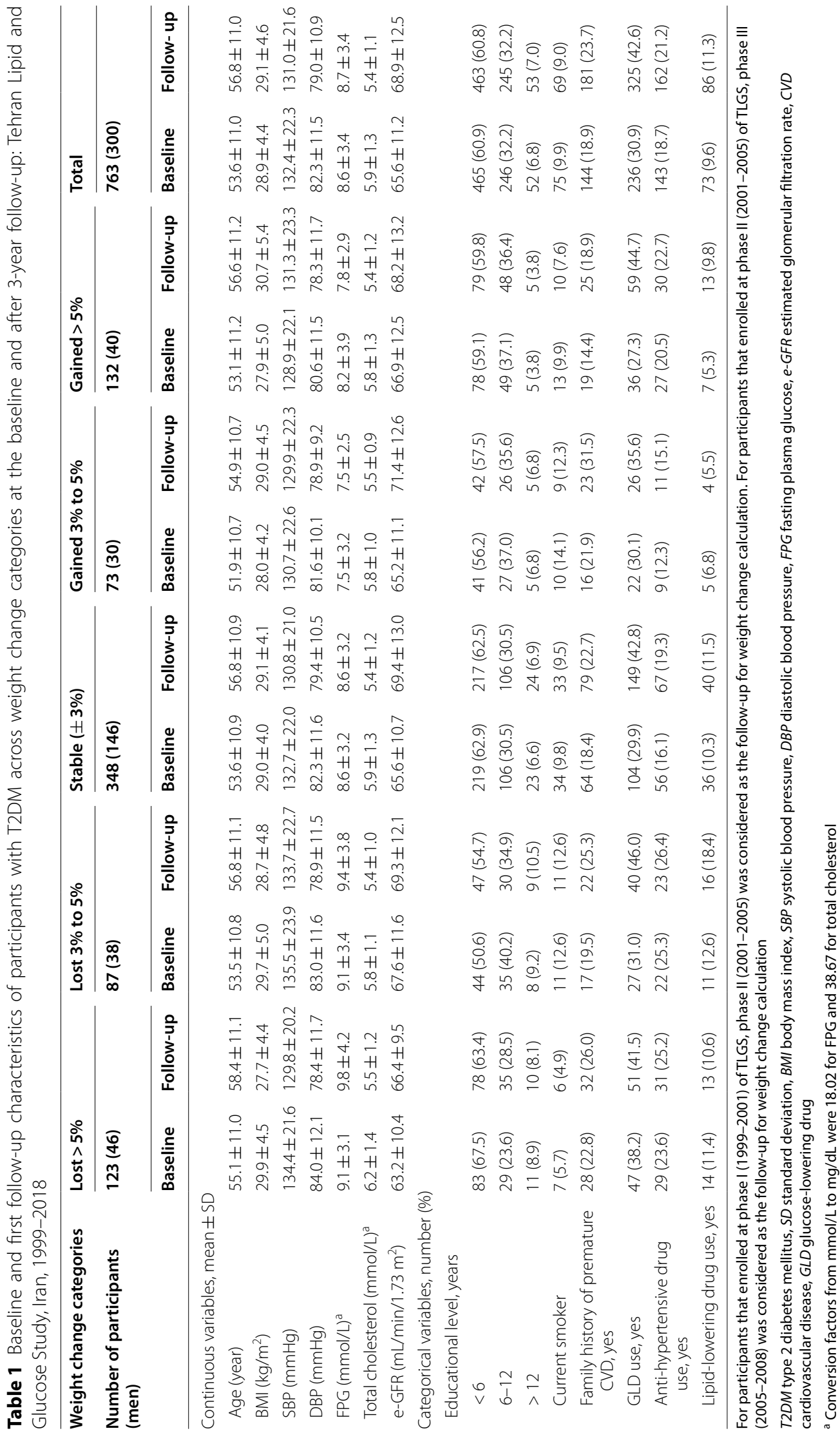


Table 2 Multivariable hazard ratios (HR) and 95\% confidence intervals (Cl) of association between weight change categories and incident CVD: Tehran Lipid and Glucose Study, Iran, 1999-2018

\begin{tabular}{|c|c|c|c|c|}
\hline & \multicolumn{2}{|l|}{ Model 1} & \multicolumn{2}{|l|}{ Model 2} \\
\hline & $\mathrm{HR}(95 \% \mathrm{Cl})$ & P-value & $\mathrm{HR}(95 \% \mathrm{Cl})$ & P-value \\
\hline \multicolumn{5}{|l|}{ Weight change categories } \\
\hline Lost $>5 \%$ & $1.18(0.84-1.65)$ & 0.333 & $1.11(0.79-1.56)$ & 0.538 \\
\hline Lost $3 \%$ to $5 \%$ & $1.04(0.70-1.53)$ & 0.863 & $0.89(0.60-1.33)$ & 0.571 \\
\hline Stable $( \pm 3 \%)$ & Reference & & Reference & \\
\hline Gained 3\% to 5\% & $0.69(0.42-1.11)$ & 0.128 & $0.76(0.46-1.23)$ & 0.260 \\
\hline Gained > 5\% & $0.71(0.49-1.03)$ & 0.075 & $0.70(0.48-1.01)$ & 0.058 \\
\hline Age, year & $1.05(1.04-1.06)$ & $<0.001$ & $1.05(1.03-1.07)$ & $<0.001$ \\
\hline Women (men as reference) & $0.90(0.70-1.15)$ & 0.394 & $0.78(0.58-1.05)$ & 0.101 \\
\hline $\mathrm{BMI}, \mathrm{kg} / \mathrm{m}^{2}$ & & & $1.00(0.97-1.03)$ & 0.894 \\
\hline \multicolumn{5}{|l|}{ Educational level, years } \\
\hline$>12$ & & & Reference & \\
\hline $6-12$ & & & $0.81(0.47-1.38)$ & 0.437 \\
\hline$<6$ & & & $0.82(0.48-1.39)$ & 0.459 \\
\hline Current smoker, yes & & & $1.64(1.03-2.61)$ & 0.036 \\
\hline GLD use, yes & & & $1.62(1.21-2.16)$ & 0.001 \\
\hline Family history of premature CVD, yes & & & $1.15(0.84-1.57)$ & 0.389 \\
\hline Hypertension, yes & & & $1.73(1.32-2.26)$ & $<0.001$ \\
\hline Hypercholesterolemia, yes & & & $1.77(1.27-2.48)$ & 0.001 \\
\hline$C K D$, yes & & & $0.78(0.58-1.05)$ & 0.097 \\
\hline $\mathrm{FPG}, \mathrm{mmol} / \mathrm{L}$ & & & $1.04(1.00-1.08)$ & 0.039 \\
\hline
\end{tabular}

Model 1: adjusted for age and sex. Model 2: Model 1 + further adjusted for BMI, educational level, current smoking (at first follow-up), GLD use (at baseline or first follow-up), family history of premature CVD, hypertension, hypercholesterolemia, CKD, and FPG

CVD cardiovascular disease, BMI body mass index, GLD glucose-lowering drugs, CKD chronic kidney disease, FPG fasting plasma glucose

controlling for the potential confounding factors in model 2, these risks became 0.70 [0.48-1.01; P-value: 0.058] for CVD and 0.61 [0.40-0.93] for CHD. Moreover, after adjustment for FPG change during the first 3 years of follow-up, the effect size of weight gain $>5 \%$ was attenuated to 0.75 [0.51-1.10; P-value: 0.138$]$ and 0.66 [043-1.01; P-value: 0.053] for incident CVD and CHD, respectively. Generally, older age, current smoking, GLD use, hypertension, hypercholesterolemia, and FPG were associated with higher risk of incident CVD/CHD in the model 2.

To show the robustness of our findings, a series of sensitivity analyses was performed. First, as presented in Additional file 2: Table S2 and Additional file 3: Table S3, based on our results from imputed baseline missing data for covariates (participant number: 1104) with the occurrence of 341 cases of incident CVD (286 CHD cases), our findings were consistent with the main analysis. Accordingly, more than $5 \%$ weight gain decreased the risk of incident CVD and CHD in model 2 with HRs of 0.69 [0.49-0.96] and 0.62 [0.43-0.90], respectively. After adjustment for FPG change, the HRs of weight gain $>5 \%$ were attenuated to $0.76[0.54-1.05$;
P-value: 0.100] and 0.67 [0.46-0.98]. Second, multivariable $\mathrm{HRs}$ and $95 \% \mathrm{CIs}$ for different weight change categories, stratified by age, sex, BMI, and GLD use, are shown in Figs. 3 and 4. Considering age stratification, for those aged $<60$ years, no significant difference was found between weight change categories; however, among participants aged $\geq 60$ years, more than 5\% weight gain had HRs of 0.59 [0.33-1.04; P-value: 0.069] and 0.36 [0.17-0.78] for incident CVD and CHD, respectively. Moreover, weight loss $>5 \%$ showed higher risk among older and lower risk among younger participants; however, it did not reach a significant level (P-value for the interaction of age $<0.2$ ). Moreover, although the interactions of weight change categories with sex, BMI groups, and GLD use were not significant, weight gain $>5 \%$ among men and weight gain $>3 \%$ among GLD users also had significantly lower risk of CVD/CHD. Third, since sulfonylurea was the most commonly used GLD in our population, we examined the association between weight change categories with incident CVD/CHD among users of sulfonylureas (Fig. 5). Those with weight gain of over 5\% were at lower risk of incident CVD and CHD. Moreover, 
Table 3 Multivariable hazard ratios (HR) and 95\% confidence intervals (Cl) of association between weight change categories and incident CHD: Tehran Lipid and Glucose Study, Iran, 1999-2018

\begin{tabular}{|c|c|c|c|c|}
\hline & \multicolumn{2}{|l|}{ Model 1} & \multicolumn{2}{|l|}{ Model 2} \\
\hline & $\mathrm{HR}(95 \% \mathrm{Cl})$ & P-value & $\mathrm{HR}(95 \% \mathrm{Cl})$ & P-value \\
\hline \multicolumn{5}{|l|}{ Weight change categories } \\
\hline Lost $>5 \%$ & $1.19(0.83-1.71)$ & 0.334 & $1.14(0.79-1.65)$ & 0.471 \\
\hline Lost $3 \%$ to $5 \%$ & $0.86(0.55-1.36)$ & 0.526 & $0.76(0.48-1.20)$ & 0.238 \\
\hline Stable ( $\pm 3 \%)$ & Reference & & Reference & \\
\hline Gained 3\% to 5\% & $0.63(0.37-1.08)$ & 0.096 & $0.66(0.38-1.15)$ & 0.141 \\
\hline Gained $>5 \%$ & $0.62(0.41-0.94)$ & 0.026 & $0.61(0.40-0.93)$ & 0.021 \\
\hline Age, year & $1.04(1.03-1.05)$ & $<0.001$ & $1.04(1.02-1.05)$ & $<0.001$ \\
\hline Women (men as reference) & $0.92(0.70-1.22)$ & 0.569 & $0.82(0.59-1.13)$ & 0.216 \\
\hline $\mathrm{BMI}, \mathrm{kg} / \mathrm{m}^{2}$ & & & $0.99(0.96-1.02)$ & 0.423 \\
\hline \multicolumn{5}{|l|}{ Educational level, years } \\
\hline$>12$ & & & Reference & \\
\hline $6-12$ & & & $0.91(0.50-1.66)$ & 0.768 \\
\hline$<6$ & & & $0.92(0.51-1.69)$ & 0.796 \\
\hline Current smoker, yes & & & $1.73(1.06-2.83)$ & 0.028 \\
\hline GLD use, yes & & & $1.75(1.27-2.41)$ & 0.001 \\
\hline Family history of premature CVD, yes & & & $1.08(0.76-1.52)$ & 0.672 \\
\hline Hypertension, yes & & & $1.63(1.22-2.19)$ & 0.001 \\
\hline Hypercholesterolemia, yes & & & $2.09(1.43-3.05)$ & $<0.001$ \\
\hline$C K D$, yes & & & $0.76(0.55-1.05)$ & 0.099 \\
\hline $\mathrm{FPG}, \mathrm{mmol} / \mathrm{L}$ & & & $1.03(0.99-1.08)$ & 0.139 \\
\hline
\end{tabular}

Model 1: adjusted for age and sex. Model 2: Model 1 + further adjusted for BMI, educational level, current smoking (at first follow-up), GLD use (at baseline or first follow-up), family history of premature CVD, hypertension, hypercholesterolemia, CKD, and FPG

CHD coronary heart disease, BMI body mass index, GLD glucose-lowering drugs, CVD cardiovascular disease, CKD chronic kidney disease, FPG fasting plasma glucose

participants with weight gain of $3-5 \%$ had a marginally significant HR of 0.37 [0.13-1.04; P-value: 0.059] for incident CHD.

\section{Discussion}

In this cohort study, during a long-term follow-up, after adjustment for well-known CVD risk factors, more than $5 \%$ weight gain within 3 years was associated with reduced risk of CVD/CHD development among an Iranian population with T2DM. However, after adjustment for FPG change, the effect sizes of the associations were attenuated to some degree but remained significant for CHD. We also found significant effect modification of age in the association between weight change and incident CVD/CHD; weight gain $>5 \%$ reduced the risk of incident CHD significantly only among those older than 60 years. Although there is no significant interaction between weight change categories and GLD use, over 3\% weight gain reduced the risk of incident CVD/CHD among GLD users.

It should be noted that previous studies with similar aims to ours calculated weight or BMI change in different time intervals and categorizations. These studies also varied in study design, study setting (clinical-based [15, 19] versus population-based $[17,26])$, and other aspects of methodology. Therefore, it is difficult to compare our findings with other studies in this field.

In contrast to our results, a flat V-shaped association was observed between BMI change and cardiac death or non-fatal MI in the Action to Control Cardiovascular Risk in Diabetes (ACCORD) study, in which stable weight had the lowest risk; the risk increased slowly as the weight was either increased or decreased; however, there is no significant difference between BMI change categories and the risk of these outcomes [19]. Moreover, among Swedish subjects with T2DM, an increased BMI during 18 months led to higher risks of CVD mortality (HR: 1.63 [1.11-2.39]) [20].

Similar to our results, data from the ORIGIN trial showed that among participants with T2DM or prediabetes, 1-year weight gain and weight loss, respectively, were associated with decreased and increased risk of an outcome composite of the first occurrence of cardiovascular death, non-fatal myocardial infarction, or non-fatal stroke during over 6 years of follow-up [15]. In a meta-analysis of prospective studies, despite a high 


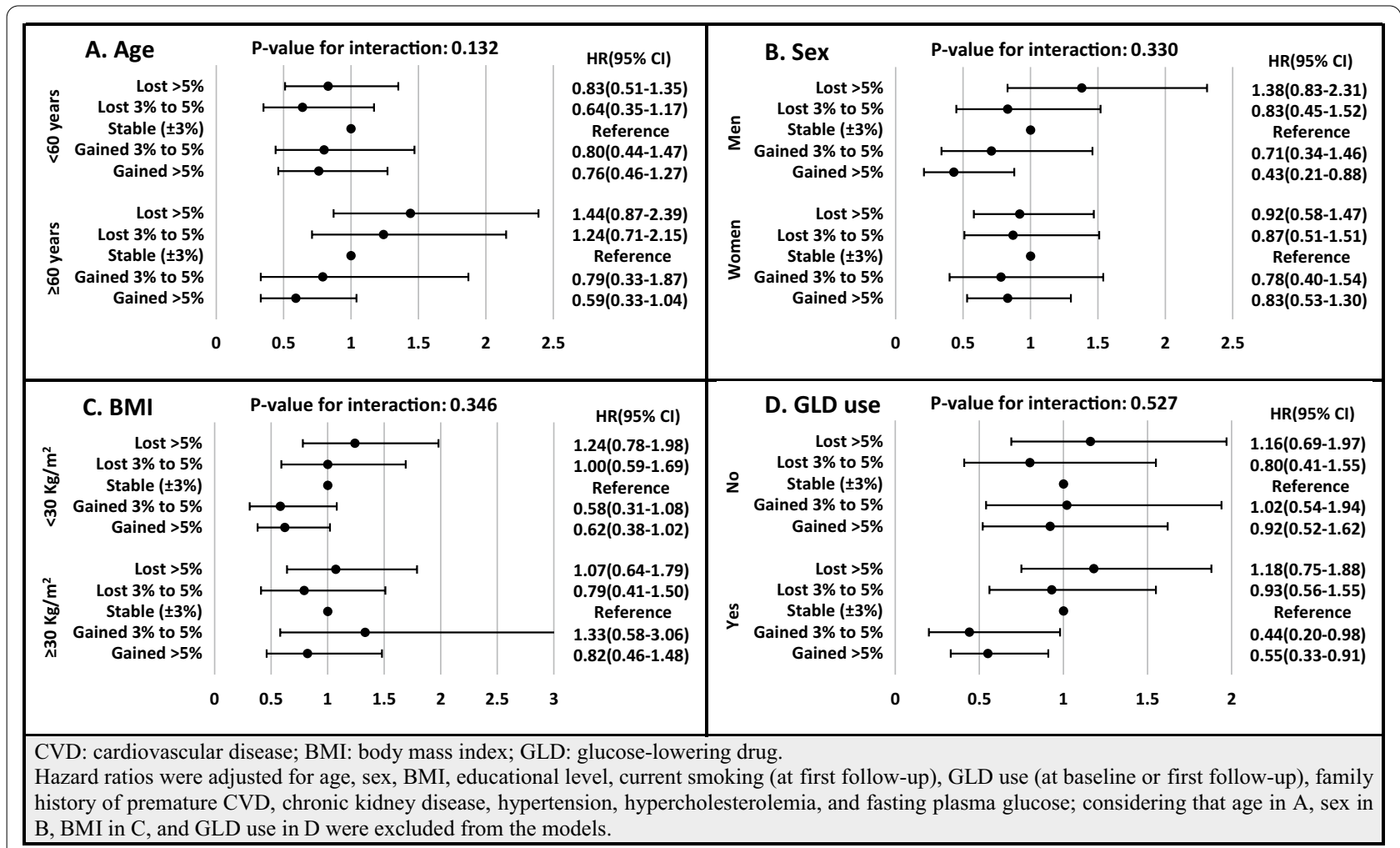

Fig. 3 Multivariable hazard ratios (HR) and 95\% confidence intervals (Cl) of association between weight change categories and incident CVD, stratified by age (A), sex (B), BMI (C), and GLD use (D): Tehran Lipid and Glucose Study, Iran, 1999-2018

heterogeneity, weight loss increased the risk of CVD mortality by $15 \%$ among overweight or obese patients with diabetes; moreover, weight gain had a HR of 0.97 [0.931.01] [13]. Based on results from the ADVANCE trial, compared to a stable weight, 2-year weight loss $>10 \%$ was associated significantly with increased risk of major cardiovascular events and CVD mortality; in contrast, weight gain did not contain a significant effect on CVD [16]. Recently, among about 20,000 White British UK biobank participants with T2DM, it was shown that the minimum mortality risk was seen in those with a BMI of $32 \mathrm{~kg} / \mathrm{m}^{2}$; moreover, using Mendelian randomization, the researchers did not find a significant impact of obesity on mortality in this population [27]. Generally, for weight gain, we did not find an unfavorable impact on CVD/ CHD among subjects with T2DM in TLGS, and over $5 \%$ weight gain was significantly associated with lower risk of incident CVD and CHD. However, our results should not translate into a recommendation for weight gain among individuals with T2DM. Our study was an observational study, and there was no intervention for intentional weight change (gain or loss). Despite this fact, it seems that weight management among diabetic individuals remained challenging. Focusing on intentional weight change among overweight or obese patients with
T2DM, based on data from the Look AHEAD (Action for Health in Diabetes) study, which compared the effect of intensive lifestyle intervention with a control group that received diabetes support and education, it was shown that 1-year weight loss was associated with significant improvements in CVD risk factors, with greater benefits in a larger range of weight loss and more prominent in the intervention group [28]; however, this intensive lifestyle intervention did not reduce the risk of incident CVD [7]. Moreover, results for the effect of bariatric surgery among obese individuals with T2DM have consistently shown improvement in cardiovascular risk factors; however, randomized controlled trials did not have adequate power to assess improvement in CVD outcomes [29].

In the current study, although the interaction between GLD usage and weight change categories was not significant, the effect of weight gain on CVD development was more prominent among GLDs users. In our data set, GLD users, mainly included users of sulfonylureas at the recruitment time, had a higher risk for incident CVD/ CHD (Tables 2 and 3); however, considering the lack of data on the duration of diabetes for our participants, this finding cannot be conclusively translated into the adverse effect of sulfonylureas or other types of GLDs on incident 


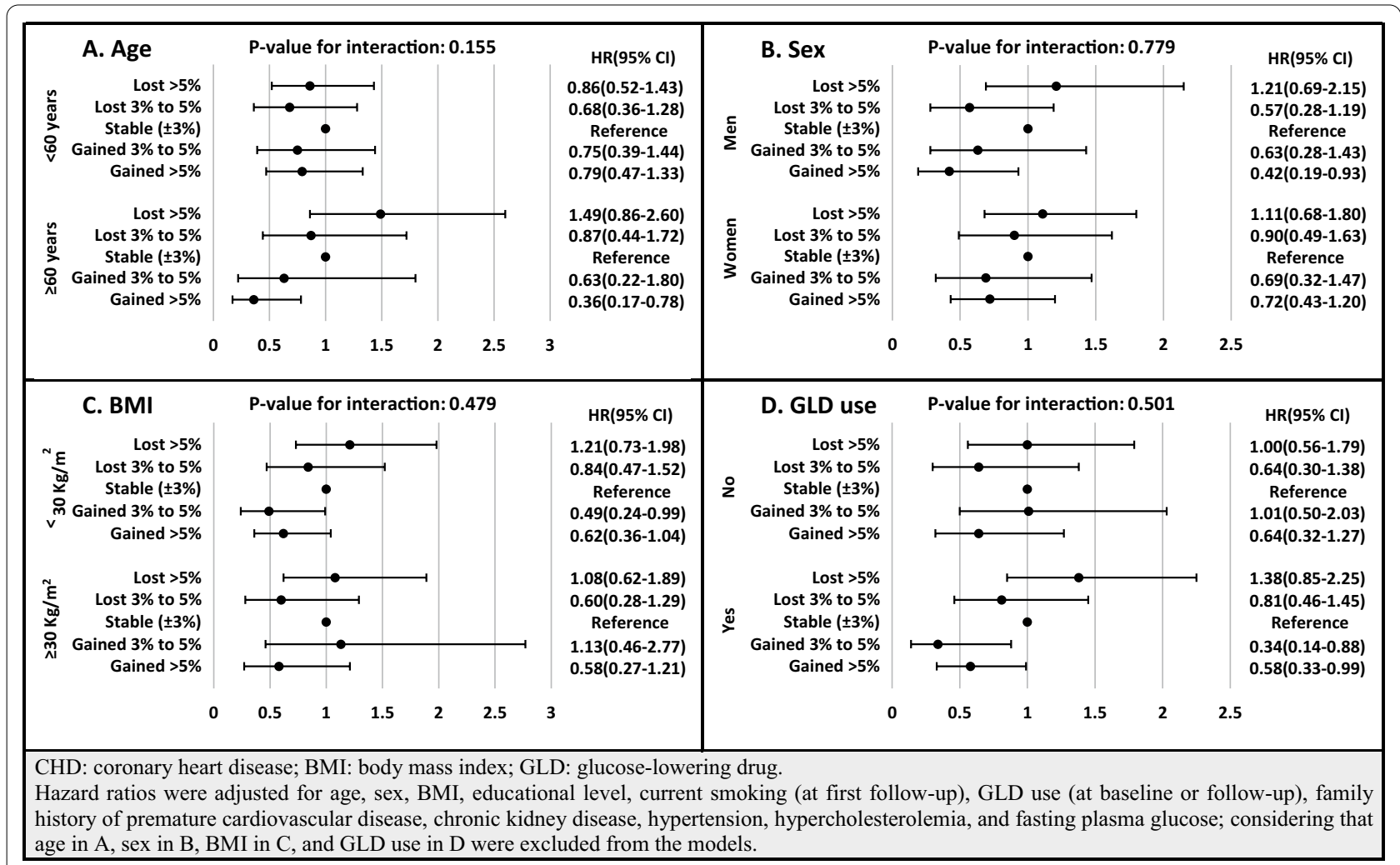

Fig. 4 Multivariable hazard ratios (HR) and 95\% confidence intervals (Cl) of association between weight change categories and incident $\mathrm{CHD}$, stratified by age (A), sex (B), BMI (C), and GLD use (D): Tehran Lipid and Glucose Study, Iran, 1999-2018

CVD/CHD, because our GLD users were known-diabetic patients with potentially more prolonged duration of disease and worse baseline characteristics rather than not GLD users (allocation bias) [30]. Furthermore, due to weight gain and hypoglycemia induced by sulfonylureas, there is still a debate about their long-term cardiovascular safety [30-33]; however, it is still recommended to clinicians to continue prescription of low-cost sulfonylureas in T2DM, with confidence in their effectiveness for prevention of microvascular complications and their potential cardiovascular safety [30, 34]. Now our findings reduced this concern about sulfonylurea-induced weight gain by showing that weight gain $>5 \%$ not only did not increase the risk of incident CVD/CHD but also it could decrease the risk among our sulfonylurea users. Similar to our finding for sulfonylureas, other previous studies showed no adverse effect on CVD outcomes for insulin- and pioglitazone-induced weight gain [35, 36]. The main explanation for this risk-reducing effect of weight gain among GLDs users might be that weight gain can be an indicator of improved patients' compliance, good response to management, and preserved beta-cell function [37]. In support of this idea, in our data analysis, after adjustment for FPG change, the HRs of the association between weight gain and risk of CVD/ CHD were attenuated, especially for incident CVD. It means that FPG change played the role of mediator for this association. Similarly, among Iranian patients with T2DM that mostly had been on GLDs, Janghorbani et al. also found weight gain to be accompanied by decreased FPG and glycosylated hemoglobin (HbA1c), which reflect improved glycemic control [38]. Importantly, new GLDs, especially analog GLP-1 and SGLT2i, have evidencebased recommendations for the managements of T2DM; however, as emphasized in different guidelines (such as American Diabetes Association), in situations that "cost is a major issue", metformin, and sulfonylureas still can be considered as a first-line therapy [34]. Moreover, in our country, the new medications are not covered by insurance companies, and only a limited number of Iranians with T2DM can use them now. Moreover, in our data set, we reassessed the situation of GLDs in 2015-2018 (phase VI of TLGS) and found that 41\% of GLDs users still took sulfonylureas (data not shown).

On the other hand, among our new cases of T2DM (those diagnosed by screening at baseline and had not used GLDs), no weight change category was related to incident CVD/CHD. It should be noted that at the 


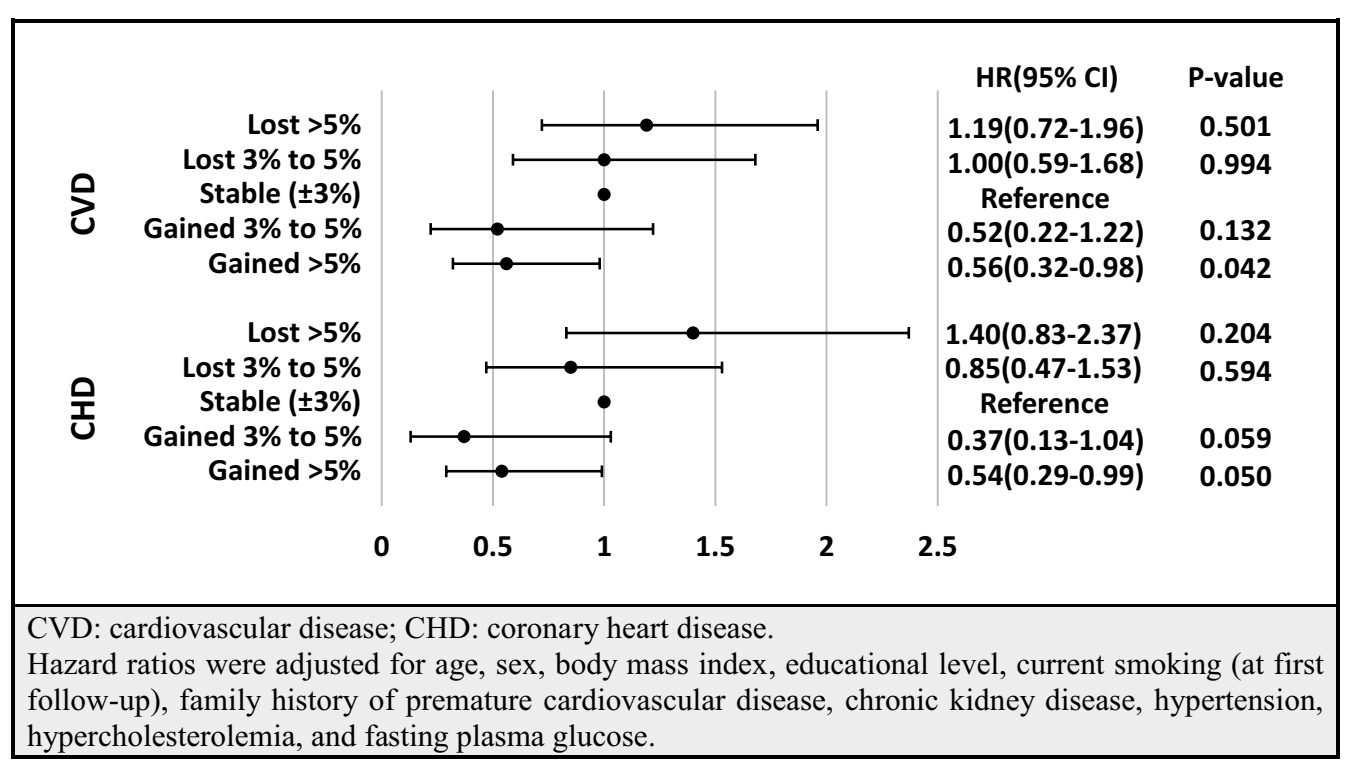

Fig. 5 Multivariable hazard ratios (HR) and 95\% confidence intervals (Cl) of association between weight change categories and incident CVD/CHD among sulfonylurea users: Tehran Lipid and Glucose Study, Iran, 1999-2018

baseline requirement, more than half of our diabetic population were newly diagnosed cases that were screened by FPG or 2h-PCPG. Despite this, we acknowledged that diabetes awareness in our population was poor at enrollment, which is consistent with previous reports in Iran $[39,40]$. Focusing on newly-diagnosed cases of T2DM, result from observational analysis of the ADDITIONEurope trial also showed that there is no evidence of a significant association between 1-year and 5-year weight change categories and incident CVD [18]; however, considering data from Cambridge center, it was shown that in addition to the significant protective effect of weight loss $\geq 5 \%$, weight gain $>2 \%$ also had a suggestive (not statistically significant) protective association with incident CVD, during 10 years of follow-up [14]. Additionally, among Scottish and German newly-diagnosed T2DM cases, respectively, weight change and BMI change were not related to macrovascular outcomes [26, 41]. A study among Korean participants also reported that a 2-year weight gain of $\geq 10 \%$ after the diagnosis of T2DM could not affect the risk of MI; however, it was introduced as a risk factor for stroke (HR:1.47 [1.20, 1.79] in the fulladjusted model) [17].

In our data analysis, not only $>5 \%$ weight gain was strongly associated with reduced risk of incident CHD among adults older than 60 years, but also weight loss $>5 \%$ showed a suggestive increased risk of incident CVD/CHD among the older-aged group only. Similarly, the impact of obesity on CVD was also different between younger and older adults in some other studies [42, 43], in which the unfavorable impact of obesity attenuated in older groups. Moreover, in contrast to younger adults, it is not clear that obese older adults should be recommended for losing their weight (intentional weight loss) $[6,44]$. Unintentional weight loss among older adults could also reflect an advanced or chronic illness, which caused a loss of muscle mass and strength (sarcopenia) [45], a condition that can be an independent risk factor for CVD development [46]. Conversely, weight gain may be a feature of neutralizing catabolic dominance and restored anabolic activity.

The current study has several strengths. First, most studies related to the effects of weight change were based on self-reported questionnaires, which may have a recall information bias; however, our study used actual measurements based on the physical examination during every follow-up. Second, most previous studies that have a similar object with us considered prevalent CVD at baseline as a covariate $[15,16,19]$, but we excluded them, and our outcome was the first occurrence of CVD events. We also had several limitations in our study. First, intentional weight loss was suggested to be associated with a lower CVD incidence rate $[47,48]$; however, as the TLGS protocol did not include any specific weight change intervention for weight modification, we did not know whether weight changes of our participants were intentional or unintentional. Second, measures of weight change do not differentiate between changes in lean or fat mass; decreased muscle mass (sarcopenia) has an 
association with higher risk for mortality and CVD [46, 49]. Third, we did not have data on the duration of diabetes; however, more than half of our diabetic population were newly diagnosed ones. Fourth, considering the cost and lack of precise method, the HbA1C was not measured for participants; however, we adjusted our models for FPG as surrogates of HbA1C level [50]. Fifth, different tools were used for physical activity level assessment in phases I (Lipid Research Clinic questionnaire) and II (Modifiable Activity Questionnaire) [21]. Therefore, we were not able to consider physical activity and its change as covariates. Sixth, variables were considered at the baseline phases (or first follow-up), and possible changes were not taken into account from baseline up to incident CVD/CHD. Seventh, because of limited outcome numbers, we were not able to examine the association of weight change categories with different subtypes of CVD, including MI, heart failure, and stroke, separately. Finally, our study population is limited to residents of Tehran, a metropolitan city, with uniform ethnicity; hence our findings may not be generalizable to rural populations or other ethnicities.

\section{Conclusion}

Our results with a long term follow-up showed that compare to stable weight $( \pm 3 \%)$, 3-year weight gain $>5 \%$ can be associated with better CVD/CHD outcomes among Iranian participants with T2DM, especially those who were older. Our findings shed light on the heterogeneous effect of weight change on CVD/CHD events among subjects with T2DM. Further researches, especially clinical trials, are needed to examine the effect of intentional and unintentional weight change on common adverse outcomes among diabetic populations. Moreover, despite an already existed concern about the effect of sulfonylureas on weight gain, we did not find an unfavorable impact on CVD events for sulfonylurea-induced weight gain.

\footnotetext{
Abbreviations

MENA: Middle East and North Africa; T2DM: Type 2 diabetes mellitus; CVD: Cardiovascular disease; GLD: Glucose-lowering drug; ADDITION: Anglo-Danish-Dutch Study of Intensive Treatment in People with Screen-Detected Diabetes in Primary Care; ADVANCE: Action in Diabetes and Vascular disease: PreterAx and diamicroN-MR Controlled Evaluation; CHD: Coronary heart disease; TLGS: Tehran Lipid and Glucose Study; BMI: Body mass index; SBP: Systolic blood pressure; DBP: Diastolic blood pressure; 2h-PCPG: 2-Hour post-challenge plasma glucose; FPG: Fasting plasma glucose; GFR: Glomerular filtration rate; MDRD: Modification of Diet in Renal Disease; CKD: Chronic kidney disease; MI: Myocardial infarction; ECG: Electrocardiogram; SD: Standard deviation; HR: Hazard ratios; Cl: Confidence interval; PMM: Predictive mean matching; ACCORD: Action to Control Cardiovascular Risk in Diabetes; HbA1c: Glycosylated hemoglobin.
}

\section{Supplementary Information}

The online version contains supplementary material available at https://doi. org/10.1186/s12933-021-01326-2.

Additional file 1: Table S1. Baseline characteristics of the respondents (study participants) and non-respondents: Tehran Lipid and Glucose Study, Iran, 1999-2018.

Additional file 2: Table S2. Multivariable hazard ratios (HR) and 95\% confidence intervals $(\mathrm{Cl})$ of association between weight change categories and incident CVD with imputed baseline missing data for covariates (number: 1104 participants): Tehran Lipid and Glucose Study, Iran, 1999-2018.

Additional file 3: Table S3. Multivariable hazard ratios (HR) and 95\% confidence intervals (Cl) of association between weight change categories and incident CHD with imputed baseline missing data for covariates (number: 1104 participants): Tehran Lipid and Glucose Study, Iran, 1999-2018.

\section{Acknowledgements}

We like to express our appreciation to the research team members and also to TLGS participants for their enthusiastic supports.

\section{Authors' contributions}

Study conception and design: SSM, RHA, MH and FH; analysis and interpretation of data: $\mathrm{MH}, \mathrm{DK}$ and $\mathrm{FH}$; drafting of the manuscript: SSM, RHA, ND, and FH; critical revision: $\mathrm{FA}$ and $\mathrm{FH}$. All authors read and approved the final manuscript.

\section{Funding}

There was not any specific source of funding for this study.

\section{Availability of data and materials}

The datasets used and analyzed during the current study are available from the corresponding author on reasonable request.

\section{Declarations}

\section{Ethics approval and consent to participate}

Written informed consent was obtained from all subjects. This study was approved by the Institutional Review Board (IRB) of the Research Institute for Endocrine Sciences, Shahid Beheshti University of Medical Science.

\section{Consent for publication}

All authors have declared their consents for publication.

\section{Competing interests}

There was no competing interest in the current study.

\section{Author details}

${ }^{1}$ Prevention of Metabolic Disorders Research Center, Research Institute for Endocrine Sciences, Shahid Beheshti University of Medical Sciences, No. 24, Parvaneh Street, Velenjak, P.O. Box: 19395-4763, Tehran, Iran. ${ }^{2}$ Student Research Committee, School of Medicine, Shahid Beheshti University of Medical Science, Tehran, Iran. ${ }^{3}$ Department of Epidemiology and Biostatistics, Research Institute for Endocrine Sciences, Shahid Beheshti University of Medical Sciences, Tehran, Iran. ${ }^{4}$ Endocrine Research Center, Research Institute for Endocrine Sciences, Shahid Beheshti University of Medical Sciences, Tehran, Iran.

Received: 24 January 2021 Accepted: 28 June 2021

Published online: 12 July 2021

References

1. Azizi F, Hadaegh F, Hosseinpanah F, Mirmiran P, Amouzegar A, Abdi $H$, Asghari G, Parizadeh D, Montazeri SA, Lotfalian M, et al. Metabolic health in the Middle East and north Africa. Lancet Diabetes Endocrinol. 2019;7:866-79. 
2. Esteghamati A, Larijani B, Aghajani MH, Ghaemi F, Kermanchi J, Shahrami A, Saadat M, Esfahani EN, Ganji M, Noshad S. Diabetes in Iran: prospective analysis from first nationwide diabetes report of National Program for Prevention and Control of Diabetes (NPPCD-2016). Sci Rep. 2017;7(1):1-10.

3. Moazzeni SS, Ghafelehbashi H, Hasheminia M, Parizadeh D, Ghanbarian A, Azizi F, Hadaegh F. Sex-specific Prevalence of coronary heart disease among Tehranian adult population across different glycemic status: Tehran lipid and glucose study, 2008-2011. BMC Public Health. 2020;20:1-9.

4. Derakhshan A, Sardarinia M, Khalili D, Momenan AA, Azizi F, Hadaegh F. Sex specific incidence rates of type 2 diabetes and its risk factors over 9 years of follow-up: Tehran lipid and glucose study. PLOS ONE. 2014;9(7):e102563.

5. Ramezankhani A, Harati H, Bozorgmanesh M, Tohidi M, Khalili D, Azizi F, Hadaegh F. Diabetes mellitus: findings from 20 years of the Tehran lipid and glucose study. Int J Endocrinol Metab. 2018;16(4 Suppl):e84784.

6. Brown R, Kuk J. Consequences of obesity and weight loss: a devil's advocate position. Obes Rev. 2015;16(1):77-87.

7. Group LAR. Cardiovascular effects of intensive lifestyle intervention in type 2 diabetes. N Engl J Med. 2013;369(2):145.

8. Care F. Standards of medical care in diabetes 2019. Diabetes Care. 2019:42(Suppl 1):S124-38.

9. Gaddey HL, Holder K. Unintentional weight loss in older adults. Am Fam Physician. 2014;89(9):718-22.

10. Nam GE, Kim W, Han K, Lee C-W, Kwon Y, Han B, Park S, Park J-H, Kim Y-H, Kim D-H. Body weight variability and the risk of cardiovascular outcomes and mortality in patients with type 2 diabetes: a nationwide cohort study. Diabetes Care. 2020;43(9):2234-41.

11. Lee H-J, Choi E-K, Han K-D, Kim DH, Lee E, Lee S-R, Oh S, Lip GY. High variability in bodyweight is associated with an increased risk of atria fibrillation in patients with type 2 diabetes mellitus: a nationwide cohort study. Cardiovasc Diabetol. 2020;19(1):1-10.

12. Hurren KM, Dunham MW. Understanding the impact of commonly utilized, non-insulin, glucose-lowering drugs on body weight in patients with type 2 diabetes. Expert Opin Pharmacother. 2018;19(10):1087-95.

13. Chen $Y$, Yang $X$, Wang J, Li Y, Ying D, Yuan H. Weight loss increases allcause mortality in overweight or obese patients with diabetes: a metaanalysis. Medicine. 2018;97(35):e12075.

14. Strelitz J, Ahern AL, Long GH, Hare MJ, Irving G, Boothby CE, Wareham NJ, Griffin SJ. Moderate weight change following diabetes diagnosis and 10 year incidence of cardiovascular disease and mortality. Diabetologia. 2019;62(8):1391-402.

15. Doehner W, Gerstein HC, Ried J, Jung H, Asbrand C, Hess S, Anker SD. Obesity and weight loss are inversely related to mortality and cardiovascular outcome in prediabetes and type 2 diabetes: data from the ORIGIN trial. Eur Heart J. 2020;41 (28):2668-77.

16. Lee AK, Woodward M, Wang D, Ohkuma T, Warren B, Sharrett AR, Williams B, Marre M, Hamet P, Harrap S. The risks of cardiovascular disease and mortality following weight change in adults with diabetes: results from ADVANCE. J Clin Endocrinol Metab. 2020;105(1):152-62.

17. Kim MK, Han K, Koh ES, Kim ES, Lee M-K, Nam GE, Kwon H-S. Weight change and mortality and cardiovascular outcomes in patients with newonset diabetes mellitus: a nationwide cohort study. Cardiovasc Diabetol. 2019:18(1):36

18. Strelitz J, Sharp SJ, Khunti K, Vos RC, Rutten GE, Webb DR, Witte DR, Sandbæk A, Wareham NJ, Griffin SJ. Association of weight loss and weight loss maintenance following diabetes diagnosis by screening and incidence of cardiovascular disease and all-cause mortality: an observational analysis of the ADDITION-Europe trial. Diabetes Obes Metab. 2020;23(3):730-41.

19. Xing Z, Pei J, Huang J, Peng X, Chen P, Hu X, Gao S. Weight change is associated with increased all-cause mortality and non-cardiac mortality among patients with type 2 diabetes mellitus. Endocrine. 2019;64(1):82-9.

20. Bodegard J, Sundström J, Svennblad B, Östgren CJ, Nilsson P, Johansson $\mathrm{G}$. Changes in body mass index following newly diagnosed type 2 diabetes and risk of cardiovascular mortality: a cohort study of 8486 primary-care patients. Diabetes Metab. 2013;39(4):306-13.

21. Azizi F, Ghanbarian A, Momenan AA, Hadaegh F, Mirmiran P, Hedayati M, Mehrabi Y, Zahedi-AsI S. Prevention of non-communicable disease in a population in nutrition transition: Tehran lipid and glucose study phase II. Trials. 2009;10(1):5.
22. Levey AS, Coresh J, Greene T, Marsh J, Stevens LA, Kusek JW, Van Lente F, Collaboration CKDE. Expressing the modification of diet in renal disease study equation for estimating glomerular filtration rate with standardized serum creatinine values. Clin Chem. 2007;53(4):766-72.

23. Stevens J, Truesdale KP, McClain JE, Cai J. The definition of weight maintenance. Int J Obes. 2006;30(3):391-9.

24. Van Buuren S. Flexible imputation of missing data. Boca Raton: CRC Press; 2018.

25. Steyerberg EW. Clinical prediction models. Cham: Springer; 2019.

26. Aucott LS, Philip S, Avenell A, Afolabi E, Sattar N, Wild S. Patterns of weight change after the diagnosis of type 2 diabetes in Scotland and their relationship with glycaemic control, mortality and cardiovascular outcomes: a retrospective cohort study. BMJ Open. 2016;6(7):e010836.

27. Jenkins DA, Wade KH, Carslake D, Bowden J, Sattar N, Loos RJ, Timpson $\mathrm{NJ}$, Sperrin M, Rutter MK. Estimating the causal effect of BMI on mortality risk in people with heart disease, diabetes and cancer using Mendelian randomization. Int J Cardiol. 2021;330:214-20.

28. Wing RR, Lang W, Wadden TA, Safford M, Knowler WC, Bertoni AG, Hill JO, Brancati FL, Peters A, Wagenknecht L. Benefits of modest weight loss in improving cardiovascular risk factors in overweight and obese individuals with type 2 diabetes. Diabetes Care. 2011;34(7):1481-6.

29. Arnold SV, Bhatt DL, Barsness GW, Beatty AL, Deedwania PC, Inzucchi SE, Kosiborod M, Leiter LA, Lipska KJ, Newman JD. Clinical management of stable coronary artery disease in patients with type 2 diabetes mellitus: a scientific statement from the American Heart Association. Circulation. 2020;141(19):e779-806.

30. Wexler DJ. Sulfonylureas and cardiovascular safety: the final verdict? JAMA. 2019;322(12):1147-9.

31. Mannucci E, Monami M, Candido R, Pintaudi B, Targher G, Joint Panel for Italian S-A. Effect of insulin secretagogues on major cardiovascular events and all-cause mortality: a meta-analysis of randomized controlled trials. Nutr Metab Cardiovasc Dis. 2020;30(10):1601-8.

32. Powell WR, Christiansen CL, Miller DR. Meta-analysis of sulfonylurea therapy on long-term risk of mortality and cardiovascular events compared to other oral glucose-lowering treatments. Diabetes Ther Res Treat Educ Diabetes Relat Disord. 2018;9(4):1431-40.

33. Xu J, Rajaratnam R. Cardiovascular safety of non-insulin pharmacotherapy for type 2 diabetes. Cardiovasc Diabetol. 2017;16(1):18.

34. AD Association. 9. Pharmacologic approaches to glycemic treatment: standards of medical care in diabetes_-2021. Diabetes Care. 2021;44(Supplement 1):S111-24.

35. Anyanwagu U, Mamza J, Donnelly R, Idris I. Association between insulininduced weight change and CVD mortality: evidence from a historic cohort study of 18,814 patients in UK primary care. Diabetes Metab Res Rev. 2018;34(1):e2945.

36. Doehner W, Erdmann E, Cairns R, Clark AL, Dormandy JA, Ferrannini E, Anker SD. Inverse relation of body weight and weight change with mortality and morbidity in patients with type 2 diabetes and cardiovascular co-morbidity: an analysis of the PROactive study population. Int J Cardiol. 2012;162(1):20-6.

37. Davies MJ. Insulin secretagogues. Curr Med Res Opin. 2002;18(sup 1):s22-30.

38. Janghorbani M, Amini M, Salehi-Marzijarani M. Weight change, blood pressure, lipids and glycemic control among patients with type 2 diabetes. Ann Nutr Metab. 2011;58(2):141-9.

39. Esteghamati A, Gouya MM, Abbasi M, Delavari A, Alikhani S, Alaedini F, Safaie A, Forouzanfar M, Gregg EW. Prevalence of diabetes and impaired fasting glucose in the adult population of Iran: national survey of risk factors for non-communicable diseases of Iran. Diabetes Care. 2008;31(1):96-8.

40. Esteghamati A, Etemad K, Koohpayehzadeh J, Abbasi M, Meysamie A, Noshad S, Asgari F, Mousavizadeh M, Rafei A, Khajeh E. Trends in the prevalence of diabetes and impaired fasting glucose in association with obesity in Iran: 2005-2011. Diabetes Res Clin Pract. 2014;103(2):319-27.

41. Polemiti E, Baudry J, Kuxhaus O, Jäger S, Bergmann MM, Weikert C, Schulze MB. BMI and BMI change following incident type 2 diabetes and risk of microvascular and macrovascular complications: the EPIC-potsdam study. Diabetologia. 2021;64(4):814-25.

42. Canning KL, Brown RE, Jamnik VK, Kuk JL. Relationship between obesity and obesity-related morbidities weakens with aging. J Gerontol Ser A Biomed Sci Med Sci. 2014;69(1):87-92. 
43. Rovella V, Anemona L, Cardellini M, Scimeca M, Saggini A, Santeusanio G, Bonanno E, Montanaro M, Legramante IM, Ippoliti A. The role of obesity in carotid plaque instability: interaction with age, gender, and cardiovascular risk factors. Cardiovasc Diabetol. 2018;17(1):46.

44. Brown RE, Kuk JL. Age-related differences in the consequences of obesity on cardiovascular disease, type 2 diabetes, osteoarthritis, cancer, physical function, osteoporosis, cognitive function, and mortality risk in the elderly. Healthy Aging Clin Care Elder. 2014;6:25.

45. Cruz-Jentoft AJ, Sayer AA. Sarcopenia. Lancet. 2019:393(10191):2636-46.

46. Chin SO, Rhee SY, Chon S, Hwang Y-C, Jeong I-K, Oh S, Ahn KJ, Chung HY, Woo J-T, Kim S-W. Sarcopenia is independently associated with cardiovascular disease in older Korean adults: the Korea national health and nutrition examination survey (KNHANES) from 2009. PLOS ONE. 2013:8(3):e60119.

47. Williamson DF, Thompson TJ, Thun M, Flanders D, Pamuk E, Byers T. Intentional weight loss and mortality among overweight individuals with diabetes. Diabetes Care. 2000;23(10):1499-504.
48. Gregg EW, Jakicic JM, Blackburn G, Bloomquist P, Bray GA, Clark JM, Coday M, Curtis JM, Egan C, Evans M, et al. Association of the magnitude of weight loss and changes in physical fitness with long-term cardiovascular disease outcomes in overweight or obese people with type 2 diabetes: a post-hoc analysis of the look AHEAD randomised clinical trial. Lancet Diabetes Endocrinol. 2016;4(11):913-21.

49. De Stefani FDC, Pietraroia PS, Fernandes-Silva MM, Faria-Neto J, Baena CP. Observational evidence for unintentional weight loss in all-cause mortality and major cardiovascular events: a systematic review and metaanalysis. Sci Rep. 2018;8(1):1-11.

50. AD Association. 6. Glycemic targets. Diabetes Care. 2015;38(Supplement 1):S33-40.

\section{Publisher's Note}

Springer Nature remains neutral with regard to jurisdictional claims in published maps and institutional affiliations.
Ready to submit your research? Choose BMC and benefit from:

- fast, convenient online submission

- thorough peer review by experienced researchers in your field

- rapid publication on acceptance

- support for research data, including large and complex data types

- gold Open Access which fosters wider collaboration and increased citations

- maximum visibility for your research: over $100 \mathrm{M}$ website views per year

At BMC, research is always in progress.

Learn more biomedcentral.com/submissions 